trained in pleural procedures (Group B) in 32 patients. Primary endpoints were success of the pleural procedure, procedural pain using visual analogue score (VAS), patient anxiety using short form State Trait Anxiety Index (STAI). Secondary outcome measures were complications. Non parametric statistical tests were used for analysis.

Results There was no statistically significant (NS) difference between groups as assessed by primary endpoints (Table 1). There was one failure to undertake therapeutic pleural aspiration in Group B. Delayed complications were drain dislodgement in Group A and re-expansion pulmonary oedema in Group B. Conclusion We believe this is the first randomised control study to test if after appropriate training a Pleural Nurse Practitioner is able to safely and effectively undertake pleural procedures with equivalence in practice to trained doctors.

\begin{tabular}{|l|c|c|} 
& $\begin{array}{c}\text { Group A } \\
\text { (NP) }\end{array}$ & $\begin{array}{c}\text { Group B } \\
\text { (Dr) }\end{array}$ \\
\hline Pleural procedures & Total 17 & Total 15 \\
: Diagnostic pleural aspiration & 8 & 2 \\
Therapeutic pleural aspiration & 4 & 3 \\
Chest drain insertion & 3 & 6 \\
& 2 & 4 \\
\hline Pleural procedure success (\%) & & \\
\hline Procedural pain medianVAS score (range) & 100 & 93.75 \\
\hline Patient STAl (range) & $2^{*}(0-7)$ & $3^{3}(0.7)$ \\
\hline
\end{tabular}

$(=\mathrm{NS})$

Abstract S81 Figure 1. Graph representing the changes in comfort and neural respiratory drive with increasing trigger delay

\section{S82 MULTI-CENTRE PROSPECTIVE COMPARISON OF THE BTS AND ACCP GUIDELINES TO DETERMINE SIZE IN PRIMARY SPONTANEOUS PNEUMOTHORAX} ${ }^{9} \mathrm{M}$ Shittu, ${ }^{10} \mathrm{Y}$ Vali, ${ }^{7} \mathrm{~K}$ Chong, ${ }^{2} \mathrm{~A}$ Wilkinson, ${ }^{4} \mathrm{~T}$ Mikolasch, ${ }^{10} \mathrm{~S}$ Brij,,${ }^{11} \mathrm{~S}$ Jenkins, ${ }^{3} \mathrm{~A}$ Kamath, ${ }^{3} \mathrm{M}$ Pasteur, ${ }^{12} \mathrm{~J}$ Wason, ${ }^{1} \mathrm{SJ}$ Marciniak; ${ }^{1}$ Cambridge University Hospitals NHS Foundation Trust, Cambridge, United Kingdom; ${ }^{2}$ East and North Hertfordshire NHS Trust, Stevenage, United Kingdom; ${ }^{3}$ Norfolk and Norwich University Hospitals NHS Foundation Trust, Norwich, United Kingdom; ${ }^{4}$ Luton and Dunstable Hospitals NHS Foundation Trust, Luton, United Kingdom; ${ }^{5}$ Basildon and Thurrock University Hospitals NHS Foundation Trust, Basildon, United Kingdom; ' ${ }^{6}$ Queen Elizabeth Hospital King's Lynn NHS Foundation Trust, King's Lynn, United Kingdom; 'West Suffolk Hospitals NHS Foundation Trust, Bury St Edmunds, United Kingdom; ${ }^{8}$ James Paget University Hospitals NHS Foundation Trust, Great Yarmouth, United Kingdom; ${ }^{9}$ Southend University Hospitals NHS Foundation Trust, Westcliff-on-Sea, United Kingdom; ${ }^{10}$ Peterborough and Stamford Hospitals NHS Foundation Trust, Peterborough, United Kingdom; ${ }^{11}$ Mid Essex Hospital Services NHS Trust, Chelmsford, United Kingdom; ${ }^{12}$ MRC Biostatistics Unit, Institute of Public Health, University of Cambridge, Cambridge, United Kingdom

\subsection{6/thoraxjn-2013-204457.89}

Introduction and Objectives Attempts to develop standardised guidelines in the management of primary spontaneous pneumothorax (PSP) have been severely hampered by a lack of high quality clinical research. The American College of Chest Physicians (ACCP) and BTS guidelines are based on non-analytical studies and expert opinion. Remarkably, no consensus regarding the definition of PSP severity exists, with the ACCP and BTS each using different arbitrary measurements: hilar size $>2 \mathrm{~cm}$ (BTS) versus apical size $>3 \mathrm{~cm}$ (ACCP). The objective of this study is to define the critical size of PSP.
Methods A multi-centre prospective comparison of 168 consecutive patients presenting with PSP was performed in 13 NHS hospitals in the East of England over a period of 15 months. We compared the ability of the BTS and ACCP definitions to predict the eventual need for intercostal chest drain (ICD) insertion. Since current BTS guidelines state that pleural aspiration should be attempted prior to drainage in non-compromised PSP patients, we reasoned that ICD insertion was a valid endpoint. Using a logistic regression model that included hospital, age, hilar size, apical size and the hilar-apical interaction, we generated receiver operating characteristic (ROC) curves reflecting the probability of either measure correctly predicting the eventual need for ICD.

Results One hundred and sixteen of 168 patients for whom data were collected had been treated according to BTS guidelines. Of these, 39 eventually required ICD insertion. The correlation between hilar and apical distances was high (0.7). The logistic regression showed that hilar distance was statistically significant $(\mathrm{p}<0.001)$, but apical distance and the interaction were not. The sensitivity and specificity from using BTS guidelines were 0.667 (95\% CI $0.510-0.794)$ and $0.805(0.703-$ 0.878 ) respectively, whereas the same values using the ACCP guidelines were $0.948(0.831-0.986)$ and 0.351 (0.253-0.462). Conclusion Guidelines based on hilar distance, such as the BTS's, are likely to be more informative in predicting the eventual need for ICD. However, the two distances are highly correlated. This study, for the first time, provides an evidence-based clinically relevant definition of PSP requiring ICD that will guide treatment and serve as the foundation for subsequent trials.

\section{S83 THE USE OF INDWELLING PLEURAL CATHETERS (IPC) WITH OR WITHOUT CONCURRENT TALC POUDRAGE (TP) AT MEDICAL THORACOSCOPY (MT) FOR CASES OF SUSPECTED TRAPPED LUNG (TL)}

S Chatterii, T Pulimood, E Reid, J Herre, P Sivasothy; Cambridge University Hospitals Foundation Truts, Cambridge, United Kingdom

\subsection{6/thoraxjnl-2013-204457.90}

Background Recurrent symptomatic non-infectious pleural effusions can be effectively treated with MT and TP with pleurodesis rates approaching $85 \%$ in variety of malignant and benign aetiologies. When TL is likely at MT, management is uncertain.

Aim We report on a single centre experience of inserting an IPC at MT with or without talc poudrage for suspected trapped lung. TL was suspected if any of the following were observed (a) failure of lung to inflate on voluntary coughing at MT, (b) visceral involvement of more than 25\%, (c) radiological evidence of endobronchial compromise and (d) hydropneumothorax following previous thoracocentesis.

Method A review of all IPC insertions at MT performed at our institution between March 2009 and Feb 2013 assessing indications, length of stay (LoS) after procedure, use of concurrent TP, IPC removal rates and recorded complications. All cases had been performed using rigid thoracoscopy. TP was performed with $4 \mathrm{~g}$ sterile graded talc. Rocket 16F IPC were used in all cases.

Results All IPCs were inserted during MT for likely or possible TL. $\mathrm{N}=36$ cases. 14 male. Diagnoses-10 benign recurrent effusions; 26 malignant. See table 1 for details. Median age $73 \mathrm{yrs}$ (45-92). Median LoS post procedure-non-elective 7 days (123); elective 2.5 days (0-6). 22 (56\%) had concurrent TP (18 for malignancy). 14 (36\%) had their IPC removed with median time to removal 40 days (28-119) and of these, 11 had received 